\title{
A fixed point result for semigroups of monotone operators and a solution of discontinuous nonlinear functional-differential equations
}

\author{
Nabil Machrafi (1D) \\ Mohammed V University in Rabat, Faculty of Sciences, Department of Mathematics, Team GrAAF, \\ Laboratory LMSA, Center CeReMAR, B.P. 1014 RP, Rabat, Morocco
}

\begin{abstract}
We improve some fixed point theorems by stating a fixed point result for semigroups of monotone operators in the setting of ordered Banach spaces with a normal cone. We illustrate the usefulness of our results by proving the existence and conditional unicity of a solution of an initial value problem for discontinuous nonlinear functional-differential equations under natural hypotheses involving the order structure of the underlying space.
\end{abstract}

Mathematics Subject Classification (2020). 47H10, 47H07, 34K05, 65L03

Keywords. fixed point, semitopological semigroup, ordered Banach space, normal cone, functional-differential equation, lower and upper solutions

\section{Introduction}

Since semigroups of self-mappings generalize powers of a self-mapping, it is natural to study their fixed points using the well-known technique of applying a contracting mapping principle to some power of that self-mapping. We will, in this paper, use the following generalized version of Banach contraction principle in the framework of partially ordered metric spaces; see also [13, Th. 2.1] for the first result given in this direction.

Theorem 1.1 ([12, Theorems 2.2-2.5]). Let $(X, d)$ be a complete metric space endowed with a partial ordering $\leq$. Let $T: X \rightarrow X$ be a nondecreasing (order-preserving) mapping with the contraction condition

$$
\exists k \in(0,1) \quad \forall x, y \in X \quad(x \leq y \Rightarrow d(T x, T y) \leq k d(x, y)) .
$$

Assume that $(X, d, \leq)$ is such that one of the the following conditions holds:

for any nondecreasing sequence $\left(x_{n}\right) \subset X$, if $x_{n} \rightarrow x$ in $X$, then $x_{n} \leq x \forall n \in \mathbb{N}$, and there exists $x_{0} \in X$ with $x_{0} \leq T x_{0}$;

for any nonincreasing sequence $\left(x_{n}\right) \subset X$, if $x_{n} \rightarrow x$ in $X$, then $x \leq x_{n} \forall n \in \mathbb{N}$, and there exists $x_{0} \in X$ with $T x_{0} \leq x_{0}$.

Email address: n.machrafi@um5s.net.ma, nmachrafi@gmail.com

Received: 16.09.2019; Accepted: 29.05.2020 
Assume furthermore that every pair of elements of $X$ has a lower or an upper bound. Then, $T$ has a unique fixed point $x^{*}$ in $X$ and the iterative sequence $\left(T^{n} x\right)$ converges to $x^{*}$ for every $x \in X$.

Conditions (1.2) and (1.3) hold in the setting of ordered Banach spaces $E$, in which we will improve the following two known fixed point theorems when we restrict our attention to monotone operators $T$ on a closed set $C \subset E$ (this is so common since we deal in this case with operators preserving the order structure) with a lower (resp. upper) fixed point, i.e., $x_{0} \in C$ with $x_{0} \leq T x_{0}$ (resp. $T x_{0} \leq x_{0}$ ). Fixed point results for operators having lower or upper fixed points were considered in the literature to solve ordinary as well as functional-differential equations with lower or upper solutions; see for instance $[6,8,10,12]$.

Theorem 1.2 ([15, Theorem 1], [16, Theorem 1.2.12 ]). Let (E, $\|\|$.$) be a (real) Banach$ space with a transitive binary relation $\prec$ and a mapping $m: E \rightarrow E$ satisfying the following conditions:

(1) $\theta \prec m(x), x \in E$ and $\theta$ denotes the zero element in $E$.

(2) $\|m(x)\|=\|x\|, x \in E$.

Furthermore, assume that the norm on $E$ is monotone, that is

$$
\theta \prec x \prec y \Rightarrow\|x\| \leq\|y\|, \quad x, y \in E .
$$

Let the operator $T: E \rightarrow E$ be given with the following contraction condition:

$$
m(T x-T y) \prec A m(x-y), x, y \in E
$$

for some bounded linear operator $A$ on $E$ with the following properties:

(3) $\theta \prec x \prec y \Rightarrow A x \prec A y$.

(4) $r(A)<1$, where $r(A)$ stands for the spectral radius of $A$.

Then, $T$ has a unique fixed point $x^{*}$ in $E$ and the iterative sequence $\left(T^{n} x\right)$ converges to $x^{*}$ for every $x \in E$.

Theorem 1.3 ([8, Theorem 3.1.14]). Let $E$ be an ordered Banach space with a normal generating cone $E^{+}$and $T: E \rightarrow E$ be an operator. If there exists a positive linear bounded operator $A: E \rightarrow E,\|A\|<1$ such that

$$
-A(x-y) \leq T x-T y \leq A(x-y), \quad x, y \in E, y \leq x,
$$

then $T$ has a unique fixed point $x^{*}$ in $E$ and the iterative sequence $\left(T^{n} x\right)$ converges to $x^{*}$ for every $x \in E$.

We will improve the above theorems through the followings:

- We will consider semigroups of operators instead of a single one. In this case, the notion of a lower (resp. upper) fixed point of an operator will be naturally extended to the existence of an element with a monotone orbit for that semigroup of operators.

- As a less restrictive contraction condition than (1.5) and (1.6), we will consider the following one:

$$
-A(x-y) \leq T x-T y \leq A(x-y), \quad x, y \in C, y \leq x,
$$

where $A$ is some positive bounded linear operator on $E$ with $r(A)<1$. While conditions of Theorem 1.2 and Theorem 1.3 (see for the latter theorem [8, p 118]) imply necessarily the uniform continuity of the operator $T$, such operator is not necessarily continuous under conditions of our main theorems (hence, our results are stated for discontinuous operators in general).

- Comparing (1.5) and (1.7), one observes that the structure of the underlying space is relaxed by avoiding the mapping $m$ on $E$. In this case, monotonicity of the norm of $E$, or its weak alternative, namely, the normality of the cone of $E$ will suffice to state our fixed point results. This fact is motivated by the following example from [2, Example 3]. 
Let us recall first that a cone $K$ of an ordered normed vector space $(E,\|\cdot\|, \leq)$ is said to be normal, if there exists a constant $N>0$ such that

$$
\theta \leq x \leq y \Rightarrow\|x\| \leq N\|y\|, \quad x, y \in E,
$$

equivalently, if $E$ admits an equivalent monotone norm, i.e., an equivalent norm satisfying condition (1.4) for the partial order relation of $E$; see [1, Theorem 2.38]. Moreover, $K$ is said to be generating if the vector subspace generated by $K$ coincides with $E$, i.e., $E=K-K$. Lattice cones of the classical function spaces that are Banach lattices are special examples of normal and generating cones. More details on cone theory can be found in $[1,8]$.

Example 1.4. Let $l_{2}$ be equipped with its standard inner product norm $\|\cdot\|$ and the ordering $\leq$ given by the closed positive cone,

$$
K=\left\{\left(x_{k}\right)_{k=1}^{\infty}: x_{2 k-1} \geq k x_{2 k} \geq 0 \text { for all } k\right\} .
$$

It follows from [2, Example 3] that the ordered normed vector space $E=K-K$ is a vector lattice that admits no equivalent absolute norm $\||||| \mid$ (i.e. ||||$x||||=|||x|||, x \in E$, where $|x|:=x \vee-x$ the join of $\{x,-x\}$ ), and hence no equivalent norm satisfying condition (2) of Theorem 1.2, where $m: E \rightarrow E$ is given by $m(x)=|x|$ (which is the so common case in function spaces). However, since $K$ is a subset of the standard cone $l_{2}^{+} \subset l_{2}$ with respect to which the norm $\|$.$\| is monotone, the latter is also monotone with respect to$ the cone $K$.

The last section of the paper is devoted to the application of our results in solving the order counterpart of the following initial value problem for nonlinear functional-differential equations:

$\left\{\begin{array}{l}\left.u^{\prime}(t)=f\left(t, u\left(h_{1}(t)\right), \ldots, u\left(h_{r}(t)\right), u^{\prime}(t)\right) \text { for a.e. } t \in[0, R] \text { (resp. } \forall t \in[0, R]\right) ; \\ u(0)=0,\end{array}\right.$

where $R>0$, the unknown $u$ belongs to $A C[0, R]$ (resp. $C_{1}[0, R]$ ) the space of real-valued absolutely continuous (resp. continuously differentiable) functions on $[0, R]$,

$$
\left(t, x_{1}, \ldots, x_{r+1}\right) \rightarrow f\left(t, x_{1}, \ldots, x_{r+1}\right)
$$

is a given real-valued function defined on the set $[0, R] \times \mathbb{R}^{r+1}$ and Lebesgue measurable with respect to $t$ for all $\left(x_{1}, \ldots, x_{r+1}\right) \in \mathbb{R}^{r+1}$, and $h_{i}:[0, R] \rightarrow[0, R]$ are continuous functions. This means solving Problem (1.8) under suitable hypotheses involving the order structure of the underlying space, while the same problem has been studied in $[15, \mathrm{p}$ 183] under hypotheses that do not involve this structure; see also [16, $\mathrm{p} 49]$.

The essential order-type hypothesis here is the existence of a lower or an upper solution of Problem (1.8) that will generate its solution. This problem is said to have a lower solution if there exists $u_{0} \in A C[0, R]$ (resp. $\left.C_{1}[0, R]\right)$ such that

$\left\{\begin{array}{l}u_{0}^{\prime}(t) \leq f\left(t, u_{0}\left(h_{1}(t)\right), \ldots, u_{0}\left(h_{r}(t)\right), u_{0}^{\prime}(t)\right) \text { for a.e. } t \in[0, R] \quad(\text { resp. } \forall t \in[0, R]) ; \\ u_{0}(0) \leq 0 .\end{array}\right.$

An upper solution is defined similarly with the reversed inequalities. Assuming the existence of a lower (resp. upper) solution $u_{0}$ of Problem (1.8), we are able to localize its solution in the order interval of functions satisfying $u_{0}(t) \leq u(t), t \in[0, R]$ (resp. $\left.u(t) \leq u_{0}(t), t \in[0, R]\right)$. Solutions of nonlinear integro-differential equations having a lower or an upper solution have been studied in the literature in many works; see for instance $[8,10,12]$.

Also, the assumption of continuity of the function $f$ in [15, Theorem 3$]$ is replaced here with its increasing monotonicity with respect to $\left(x_{1}, \ldots, x_{r+1}\right)$ on $\mathbb{R}^{r+1}$ (see Sec. 4). The lack of continuity in problems for nonlinear functional-differential equations may appear 
in many situations and motivations for this kind of problems which were developed in [3, Chap. 4].

As a consequence, we prove the existence of a positive solution of Problem (1.8) under some natural hypotheses. Positive solutions of nonlinear integro-differential equations have been, in their turn, studied intensively in the literature; see for instance $[4,7,11,14]$.

\section{Preliminaries}

Throughout the paper, $C$ will denote a nonempty and closed subset of a (non-trivial) ordered Banach space $E$, i.e., a real Banach space $E$ with an ordering $\leq$ induced by a closed cone in $E$ that will be denoted by $E^{+}$. The norm of $E$ will be denoted by $\|$.$\| .$ For $x \in E$, the intervals $[x),(x]$ are the closed sets defined by $[x)=\{z \in E: x \leq z\}$, $(x]=\{z \in E: z \leq x\}$. For two vectors $x, y \in E$, if $x \leq y$ or $y \leq x$ then $x$ and $y$ are said to be comparable.

The term operator on $C$ will mean a self-mapping of $C$. An operator $T$ on $C$ is said to be monotone, if it is order-preserving, i.e., for every $x, y \in C$,

$$
x \leq y \Rightarrow T x \leq T y .
$$

Note that a linear operator $A$ on $E$ is monotone if and only if $A$ is a positive operator, i.e.,

$$
\theta \leq x \Rightarrow \theta \leq A x, \quad x \in E .
$$

In the sequel, the Banach space of bounded linear operators on $E$ and the set of positive bounded linear operators on $E$ will be denoted by $B(E)$ and $B^{+}(E)$ respectively. The spectral radius of $A \in B(E)$ is defined by

$$
r(A)=\max \{|\lambda|: \lambda \in \sigma(A)\}
$$

where $\sigma(A):=\sigma\left(A_{c}\right)$ the spectrum of $A_{c}$ and $A_{c} \in B\left(E_{c}\right)$ is the complexification of $A$ defined on the complex Banach space $E_{c}$, the complexification of $E$, by

$$
A_{c}(x+i y)=A x+i A y, \quad x, y \in E .
$$

The spectral radius of $A$ is given in terms of its norm via the following formula (wellknown as Gelfand's formula):

$$
r(A)=\lim _{n \rightarrow \infty}\left\|A^{n}\right\|^{\frac{1}{n}}=\inf _{n \in \mathbb{N}}\left\|A^{n}\right\|^{\frac{1}{n}} .
$$

In the setting of ordered Banach spaces, it is more convenient to calculate the spectral radius of a positive operator $A \in B(E)$ through its local spectral radius $r(A, x)$ at some element $x \in E$. This is defined for an operator $A \in B(E)$ by

$$
r(A, x)=\limsup _{n \rightarrow \infty}\left\|A^{n} x\right\|^{\frac{1}{n}} .
$$

The details are in the following lemma which will be useful in proving some forthcoming results.

Lemma 2.1 ([5, Proposition 5]). Let the cone $E^{+}$be normal and generating, $A \in B^{+}(E)$, and $x_{0} \in E^{+} \backslash\{\theta\}$ such that $A$ is bounded from above by $x_{0}$, that is, for every $x \in E^{+}$ there is a positive number $n(x)$ with $A x \leq n(x) x_{0}$. Then, $r(A)=r\left(A, x_{0}\right)$.

Let us consider now a commutative semitopological semigroup $S$, i.e., a semigroup with a Hausdorff topology such that for each $s \in S$, the mapping $t \rightarrow s t$ is continuous from $S$ into $S$. This includes particularly the discrete case $S=(\mathbb{N} \cup\{0\},+)$. We will use the notation $s^{n}$ to mean the nth power of $s \in S$. Since $S$ is commutative, then $S$ will be directed by the binary relation $\preceq$ defined on $S$ by the following:

$$
s \preceq t \text { if }\{s\} \cup \overline{s S} \supseteq\{t\} \cup \overline{t S} .
$$

More on semitopological semigroups and their properties can be found in [9]. 
A family $\mathcal{T}=\left\{T_{i}\right\}_{i \in S}$ of operators on $C$ is said to be a semigroup if it satisfies the following:

(1) $T_{s} T_{t}=T_{s t}$ for all $s, t \in S$;

(2) the mapping $s \rightarrow T_{s} x$ is continuous from $S$ into $C$, for every $x \in C$.

For a family $\mathcal{T}=\left\{T_{i}\right\}_{i \in S}$ of operators on a nonempty set $C$, an element $x \in C$ is said to be a fixed point of $\mathcal{T}$ if it is a fixed point of $T_{i}$ for every $i \in S$, i.e., $T_{i} x=x$ for every $i \in S$.

\section{Main results}

We formulate the following lemma, generalizing the lemma in [15, p 179], that will be used in the proof of our main result. Its proof is simple and therefore omitted.

Lemma 3.1. A sufficient condition for a commuting family $\mathcal{T}=\left\{T_{i}\right\}_{i \in S}$ of operators on a nonempty set to have a unique fixed point $x^{*}$ is that $x^{*}$ is the unique fixed point of some operator from the family $T_{i_{0}}$, where $i_{0} \in S$.

Theorem 3.2. Let the cone $E^{+}$be normal, $S$ be a commutative semitopological semigroup and $\mathcal{T}=\left\{T_{s}\right\}_{s \in S}$ be a semigroup of monotone operators on $C$. Assume that

(1) there exists $s_{0} \in S$ such that $T_{s_{0}}$ satisfies the contraction condition (1.7) with respect to some operator $A \in B^{+}(E)$;

(2) there exists $x_{0} \in C$ such that its orbit $\left\{T_{s} x_{0}\right\}_{s \in S}$ is an increasing (resp. decreasing) net.

Then $\mathcal{T}$ has a unique fixed point $x^{*}$ in $C_{0}=C \cap\left[y_{0}\right)$ (resp. $C_{0}=C \cap\left(y_{0}\right]$ ), where $y_{0}=T_{s_{0}} x_{0}$. Moreover, if $C$ is bounded, then $\lim _{s}\left\|T_{s} x-x^{*}\right\|=0$ for every $x \in C, x$ and $x^{*}$ are comparable.

Proof. Assume that the net $\left\{T_{s} x_{0}\right\}_{s \in S}$ is increasing (the other case can be dealt in a similar way). Then for every $s \in S, T_{s}$ maps $C_{0}$ into itself. Indeed, since $s_{0} \preceq s s_{0}, s \in S$ and $T_{s}$ is monotone, then

$$
T_{s_{0}} x_{0} \leq T_{s s_{0}} x_{0} \leq T_{s} x,
$$

so $T_{s} x \in C_{0}$ for every $x \in C_{0}$. Now, if $x, y \in C$ with $y \leq x$, one has

$$
\theta \leq T_{s_{0}} x-T_{s_{0}} y \leq A(x-y) .
$$

Again, since $T_{s_{0}} y \leq T_{s_{0}} x$, then

$$
\theta \leq T_{s_{0}}^{2} x-T_{s_{0}}^{2} y \leq A\left(T_{s_{0}} x-T_{s_{0}} y\right) .
$$

Applying the operator $A$ to the inequality (3.1), we get

$$
\theta \leq T_{s_{0}}^{2} x-T_{s_{0}}^{2} y \leq A^{2}(x-y) .
$$

Proceeding inductively, we have

$$
\theta \leq T_{s_{0}}^{n} x-T_{s_{0}}^{n} y \leq A^{n}(x-y)
$$

for each $n \in \mathbb{N}$. Since the cone $E^{+}$is normal, we may assume that the norm $\|\cdot\|$ is monotone. It follows that

$$
\left\|T_{s_{0}}^{n} x-T_{s_{0}}^{n} y\right\| \leq\left\|A^{n}(x-y)\right\| \leq\left\|A^{n}\right\|\|x-y\|,
$$

for each $n \in \mathbb{N}$ and for every $x, y \in C$ with $y \leq x$. Since $r(A)<1$, by Gelfand's formula there exists $n_{0} \in \mathbb{N}$ such that $\left\|A^{n_{0}}\right\|<1$. Assuming $0<\left\|A^{n_{0}}\right\|<1$, then we are in position to apply Theorem 1.1 for the mapping $\left.T_{s_{0}}^{n_{0}}\right|_{C_{0}}: C_{0} \rightarrow C_{0}$ to infer that $T_{s_{0}}^{n_{0}}$ has a unique fixed point $x^{*}$ in $C_{0}$, where $C_{0}$ is endowed with the metric induced by the norm of $E$ and $y_{0} \leq T_{s_{0}}^{n_{0}} y_{0}$ (as the net $\left\{T_{s} x_{0}\right\}_{s \in S}$ is increasing). Since $T_{s}$ maps $C_{0}$ into itself for every $s \in S$, then we infer from Lemma 3.1 that $x^{*}$ is the unique fixed point of $\mathcal{T}$ in $C_{0}$. Now, if $A^{n_{0}}=0$ then it follows from (3.2) that $T_{s_{0}}^{n_{0}}$ is the constant mapping on $C_{0}$ equal to $T_{s_{0}}^{n_{0}} y_{0}$. 
Since $y_{0} \leq T_{s_{0}}^{n_{0}} y_{0}$, then clearly $T_{s_{0}}^{n_{0}} y_{0}$ is the unique fixed point of $T_{s_{0}}^{n_{0}}$ in $C_{0}$. Therefore, by the same above argument $T_{s_{0}}^{n_{0}} y_{0}$ is the unique fixed point of $\mathcal{T}$ in $C_{0}$.

Assume now that $C$ is bounded with a diameter $M \geq 0$. Let $x \in C, x$ and $x^{*}$ be comparable, $t_{0}=s_{0}^{n_{0}}$, and $k=\left\|A^{n_{0}}\right\|$. We will show that for every $\varepsilon>0$ there exists $n \in \mathbb{N}$ such that

$$
\left\|T_{t_{0}^{n} s} x-x^{*}\right\|<\varepsilon \text { for every } s \in S .
$$

Let $\varepsilon>0$ and choose $n \in \mathbb{N}$ with $k^{n} M<\varepsilon$. Since the operators of $\mathcal{T}$ are monotone, for every $s \in S$, from (3.3) one has

$$
\begin{aligned}
\left\|T_{t_{0}^{n} s} x-x^{*}\right\| & =\left\|T_{t_{0}^{n} s} x-T_{t_{0}^{n} s} x^{*}\right\| \\
& \leq k^{n}\left\|T_{s} x-T_{s} x^{*}\right\| \\
& \leq k^{n} M<\varepsilon,
\end{aligned}
$$

as desired. Now, if $s \in S$ with $t_{0}^{n} \preceq s$, then $s \in\left\{t_{0}^{n}\right\} \cup \overline{t_{0}^{n} S}$. Therefore, it suffices to show the case $s \in \overline{t_{0}^{n} S}$. Let $\left(s_{\alpha}\right) \subset S$ be a net with $\lim _{\alpha} t_{0}^{n} s_{\alpha}=s$. It follows from (3.4) and the continuity of $s \rightarrow T_{s} x$ from $S$ into $C$ that $\left\|T_{s} x-x^{*}\right\| \leq \varepsilon$, that is $\lim _{s}\left\|T_{s} x-x^{*}\right\|=0$. This ends the proof.

Remark 3.3. (1) It is easy to see that in the particular case $S=(\mathbb{N} \cup\{0\},+)$ and $T_{n}:=T^{n}, T: C \rightarrow C$ is a monotone operator, condition (2) of the above theorem is equivalent to $x_{0}$ is a lower (resp. upper) fixed point of $T$, and hence it is a natural extension of the existence of a lower (resp. upper) fixed point of a single operator to the case of a semigroup of operators.

(2) The hypothesis of boundedness in the above theorem is realised if there exist two elements $x_{0}, z_{0} \in C, x_{0} \leq z_{0}$, such that the orbits $\left\{T_{s} x_{0}\right\}_{s \in S},\left\{T_{s} z_{0}\right\}_{s \in S}$ are an increasing and a decreasing nets respectively. Indeed, by the arguments as shown before, for every $s \in S, T_{s}$ maps the (closed) order interval $\left[T_{s_{0}} x_{0}, T_{s_{0}} z_{0}\right] \cap C$ into itself, and in this case $\mathcal{T}$ has a unique fixed point $x^{*}$ in $C_{0}=C \cap\left[T_{s_{0}} x_{0}, T_{s_{0}} z_{0}\right]$. Note that each order interval $[x, y]$ of $E, x \leq y$, is bounded since the cone $E^{+}$is normal; see [1, Theorem 2.40].

As a consequence of our main theorem, taking the particular case $S=(\mathbb{N} \cup\{0\},+)$ and $T_{n}:=T^{n}, T: C \rightarrow C$, we get an improvement of Theorem 1.2 and Theorem 1.3 in case the operator $T$ is assumed to be monotone with a lower (resp. upper) fixed point.

Corollary 3.4. Let the cone $E^{+}$be normal, $T$ be a monotone operator on $C$ with a lower (resp. upper) fixed point $x_{0} \in C$. Assume that there exists a positive integer $n_{0}$ such that the power $T^{n_{0}}$ satisfies the contraction condition (1.7) with respect to some operator $A \in B^{+}(E)$. Then, $T$ has a unique fixed point $x^{*}$ in $C_{0}=C \cap\left[x_{0}\right)$ (resp. $C_{0}=C \cap\left(x_{0}\right]$ ). Moreover, if $C$ is bounded, then the iterative sequence $\left(T^{n} x\right)$ converges to $x^{*}$ for every $x \in C, x$ and $x^{*}$ are comparable.

\section{An initial value problem for functional-differential equations}

In this section, we illustrate the applicability of our results by using Corollary 3.4 to solve Problem (1.8) under some natural order-type hypotheses. So, we will assume that

$\left(H_{1}\right)$ Problem (1.8), $u \in A C[0, R]$ admits a lower solution $u_{0}$ with $u_{0}^{\prime}(t) \geq a$ for almost all $t \in[0, R]$ and for some $a \in \mathbb{R}^{+}$, and the function

$$
f\left(., u_{0}\left(h_{1}(.)\right)-u_{0}(0), \ldots, u_{0}\left(h_{r}(.)\right)-u_{0}(0), u_{0}^{\prime}(.)\right)
$$

belongs to $L_{1}[0, R]$, the Lebesgue space of real-valued integrable functions on $[0, R]$.

Moreover, the function $f$ is assumed to be increasing with respect to $\left(x_{1}, \ldots, x_{r+1}\right)$ on $\mathbb{R}^{r+1}$, that is

$\left(H_{2}\right)$ for all $\left(t, x_{1}, \ldots, x_{r+1}\right),\left(t, y_{1}, \ldots, y_{r+1}\right) \in[0, R] \times \mathbb{R}^{r+1}$ we have

$$
x_{1} \leq y_{1}, x_{2} \leq y_{2}, \ldots, x_{r+1} \leq y_{r+1} \Rightarrow f\left(t, x_{1}, \ldots, x_{r+1}\right) \leq f\left(t, y_{1}, \ldots, y_{r+1}\right) .
$$


On the other hand, the hypothesis in [15, Theorem 3] consisting of the standard Lipschitz condition of $f$

$$
\left|f\left(t, x_{1}, \ldots, x_{r+1}\right)-f\left(t, y_{1}, \ldots, y_{r+1}\right)\right| \leq \sum_{i=1}^{r+1} L_{i}(t)\left|x_{i}-y_{i}\right|
$$

for all $\left(t, x_{1}, \ldots, x_{r+1}\right),\left(t, y_{1}, \ldots, y_{r+1}\right) \in[0, R] \times \mathbb{R}^{r+1}$, the $L_{i}$ 's are continuous and positive functions on the interval $[0, R]$, will be weakened to the Lipschitz condition:

$\left(H_{3}\right)$ for all $\left(t, x_{1}, \ldots, x_{r+1}\right),\left(t, y_{1}, \ldots, y_{r+1}\right) \in[0, R] \times \mathbb{R}^{r+1}$ with $x_{1} \geq y_{1} \geq x_{0}, x_{2} \geq y_{2} \geq$ $x_{0}, \ldots, x_{r+1} \geq y_{r+1} \geq x_{0}$, we have

$$
f\left(t, x_{1}, \ldots, x_{r+1}\right)-f\left(t, y_{1}, \ldots, y_{r+1}\right) \leq \sum_{i=1}^{r+1} L_{i}(t)\left(x_{i}-y_{i}\right)
$$

where $x_{0}=\min (a, a H)$ and $H=\min _{i=1}^{r} \min _{t \in[0, R]} h_{i}(t)$.

Finally, we make the estimate $h_{i}(t) \leq t, t \in[0, R]$ satisfying by the functions $h_{i}$ in [15, Theorem 3] less restrictive. This is

$\left(H_{4}\right)$ the functions $h_{i}, L_{i}$ satisfy the estimates

(a) $h(t):=\sup _{i=1}^{r} h_{i}(t) \leq c t^{\alpha}, t \in[0, R]$, where $c>0, \alpha \in(0,1]$ are some constants;

(b) $L_{r}(1-\alpha) c^{\frac{1}{1-\alpha}}+L_{r+1}<1$ if $\alpha \neq 1$ and $L_{r+1}<1$ if $\alpha=1$ and $c \leq 1$, where $L_{r}:=\max _{i=1}^{r}\left(\max _{[0, R]} L_{i}(t)\right) r$ and $L_{r+1}:=\max _{[0, R]} L_{r+1}(t)$.

The following theorem provides a solution of Problem (1.8), $u \in A C[0, R]$ under the above-mentioned hypotheses.

Theorem 4.1. Under the hypotheses $\left(H_{1}\right)-\left(H_{4}\right)$, Problem (1.8), $u \in A C[0, R]$ has a unique solution with $u^{\prime}(t) \geq u_{0}^{\prime}(t)$ for a.e. $t \in[0, R]$ (and hence $u(t) \geq u_{0}(t), t \in[0, R]$ ).

In what follows, we let $E=L_{1}[0, R]$ be endowed with its standard norm and the ordering $\leq$ induced by the cone

$$
E^{+}=\{u: u(t) \geq 0 \text { for a.e. } t \in[0, R]\} .
$$

We will use the following lemma that provides an estimation of the spectral radius of a Voltera-type operator on $E$.

Lemma 4.2. Let $A \in B(E)$ be the operator defined by

$$
A u(t)=L \int_{0}^{h(t)} u(s) d s, \quad t \in[0, R]
$$

where $L>0$ is some constant. Then, $r(A) \leq L(1-\alpha) c^{\frac{1}{1-\alpha}}$ if $\alpha \neq 1$ and $r(A)=0$ if $\alpha=1$ and $c \leq 1$.

Proof. Let $u_{1} \in E$ be the constant function equal to 1 . Since the cone $E^{+}$is normal and generating, $A \in B^{+}(E)$ and $A u \leq L\|u\| u_{1}$ for every $u \in E^{+}$, then by Lemma 2.1 $r(A)=r\left(A, u_{1}\right)$. Now, for $t \in[0, R]$ we see from $h(t) \leq c t^{\alpha}$ that

$$
A\left(u_{1}\right)(t)=L \int_{0}^{h(t)} u_{1}(s) d s \leq L \int_{0}^{c t^{\alpha}} d s=L c t^{\alpha} .
$$

Again, we have

$$
A^{2}\left(u_{1}\right)(t)=L \int_{0}^{h(t)} A u_{1}(s) d s \leq L \int_{0}^{c t^{\alpha}} L c s^{\alpha} d s=L^{2} \frac{c^{1+\alpha+1}}{\alpha+1} t^{\alpha(\alpha+1)},
$$


and by induction, we have

$$
A^{n}\left(u_{1}\right)(t) \leq L^{n} \frac{c^{1+\alpha+1+\ldots+\alpha^{n-1}+\ldots+\alpha+1}}{(\alpha+1)\left(\alpha^{2}+\alpha+1\right) \ldots\left(\alpha^{n-1}+\ldots+\alpha+1\right)} t^{\alpha\left(\alpha^{n-1}+\ldots+\alpha+1\right)}
$$

for every $n \geq 1$. Therefore, we have

$$
\left\|A^{n}\left(u_{1}\right)\right\| \leq L^{n} \frac{c^{1+\alpha+1+\ldots+\alpha^{n-1}+\ldots+\alpha+1}}{(\alpha+1)\left(\alpha^{2}+\alpha+1\right) \ldots\left(\alpha^{n}+\ldots+\alpha+1\right)} R^{\alpha^{n}+\ldots+\alpha+1}
$$

for every $n \geq 1$. Let $a_{n}$ be the right hand side in the last inequality. If $\alpha \neq 1$, then

$$
\frac{a_{n+1}}{a_{n}}=L \frac{c^{\alpha^{n}+\ldots+\alpha+1}}{\alpha^{n+1}+\ldots+\alpha+1} R^{\alpha^{n+1}} \rightarrow L(1-\alpha) c^{\frac{1}{1-\alpha}}
$$

as $n \rightarrow \infty$, from which we get $a_{n}^{\frac{1}{n}} \rightarrow L(1-\alpha) c^{\frac{1}{1-\alpha}}$ as $n \rightarrow \infty$. Hence,

$$
r\left(A, u_{1}\right)=\limsup _{n \rightarrow \infty}\left\|A^{n}\left(u_{1}\right)\right\|^{\frac{1}{n}} \leq \lim _{n \rightarrow \infty} a_{n}^{\frac{1}{n}}=L(1-\alpha) c^{\frac{1}{1-\alpha}},
$$

as desired. Similarly, we have $r(A)=0$ if $\alpha=1$ and $c \leq 1$.

Remark 4.3. The above lemma remains similarly true in the standard Banach lattice $E=C([0, R])$ of real-valued continuous functions on $[0, R]$, where the ordering of functions is the pointwise ordering.

Proof of Theorem 4.1. It is easily shown that Problem (1.8), $u \in A C[0, R]$ and $u^{\prime}(t) \geq$ $u_{0}^{\prime}(t)$ for a.e. $t \in[0, R]$ is equivalent to the following integral-functional equation:

$$
\left\{\begin{array}{l}
z(t)=f\left(t, \int_{0}^{h_{1}(t)} z(s) d s, \int_{0}^{h_{2}(t)} z(s) d s, \ldots, \int_{0}^{h_{r}(t)} z(s) d s, z(t)\right) \\
z(t) \geq z_{0}(t), \text { for a.e. } t \in[0, R], z, z_{0} \in E
\end{array}\right.
$$

where $u(t)=\int_{0}^{t} z(s) d s$ and $u_{0}(t)=\int_{0}^{t} z_{0}(s) d s+u_{0}(0), t \in[0, R]$. Define the operator $T$ on the interval $\left[z_{0}\right)$ of $E$ by

$$
T z(t)=f\left(t, \int_{0}^{h_{1}(t)} z(s) d s, \int_{0}^{h_{2}(t)} z(s) d s, \ldots, \int_{0}^{h_{r}(t)} z(s) d s, z(t)\right), \quad t \in[0, R] .
$$

It follows easily from the hypotheses $\left(H_{1}\right)-\left(H_{3}\right)$ that $T$ is a monotone operator on $\left[z_{0}\right)$ with $z_{0}$ as a lower fixed point. Furthermore, for every $z, w \in\left[z_{0}\right)$ with $w \leq z$, from $\left(H_{3}\right)$, one has

$$
\begin{aligned}
T z(t)-T w(t) & \leq \sum_{i=1}^{r} L_{i}(t) \int_{0}^{h_{i}(t)}(z-w)(s) d s+L_{r+1}(z-w)(t) \\
& \leq L_{r} \int_{0}^{h(t)}(z-w)(s) d s+L_{r+1}(z-w)(t) \\
& =\left(A+L_{r+1} I\right)(z-w)(t),
\end{aligned}
$$

for almost all $t \in[0, R]$, where $I$ is the identity operator of $E$ and $A \in B^{+}(E)$ is the operator of Lemma 4.2 with respect to the constant $L_{r}$. Since $\sigma\left(A+L_{r+1} I\right)=\sigma(A)+$ $L_{r+1}$, it follows from Lemma 4.2 and the hypothesis $\left(H_{4}\right)$ that

$$
r\left(A+L_{r+1} I\right) \leq r(A)+L_{r+1}<1 .
$$

Therefore, applying Corollary 3.4, we see that $T$ has a unique fixed point $z \in\left[z_{0}\right)$, that is $z$ is the unique solution of (4.3). This completes the proof. 
We get as a consequence a positive solution of Problem (1.8), $u \in A C[0, R]$ under natural hypotheses.

Corollary 4.4. Assume that the hypotheses $\left(H_{2}\right),\left(H_{4}\right)$ are satisfied, that the Lipschitz condition (4.2) is satisfied for all $\left(t, x_{1}, \ldots, x_{r+1}\right),\left(t, y_{1}, \ldots, y_{r+1}\right) \in[0, R] \times \mathbb{R}_{+}^{r+1}$ with $x_{1} \geq$ $y_{1}, x_{2} \geq y_{2}, \ldots, x_{r+1} \geq y_{r+1}$, and that the function $f(., 0, \ldots, 0)$ belongs to $\left(L_{1}[0, R]\right)^{+}$. Then, Problem (1.8), $u \in A C[0, R]$ has a unique solution with a positive derivative (and hence the solution $u$ is itself positive).

Proof. It follows from the hypotheses that Problem (1.8), $u \in A C[0, R]$ has the (everywhere) null function as a lower solution. The desired conclusion follows from Theorem 4.1 .

In case the function $f$ is assumed to be continuous on $[0, R] \times \mathbb{R}^{r+1}$, we get similar results for Problem (1.8), $u \in C_{1}[0, R]$. We omit the proofs since they follow by similar arguments applied in the setting of the standard Banach lattice $E=C[0, R]$.

Theorem 4.5. Assume that $f$ is continuous on $[0, R] \times \mathbb{R}^{r+1}$, that Problem (1.8), $u \in$ $C_{1}[0, R]$ has a lower solution $u_{0}$ with $u_{0}^{\prime}(t) \geq$ a for every $t \in[0, R]$ and for some $a \in \mathbb{R}^{+}$, and that the hypotheses $\left(H_{2}\right)-\left(H_{4}\right)$ are satisfied. Then, Problem $(1.8), u \in C_{1}[0, R]$ has a unique solution with $u^{\prime}(t) \geq u_{0}^{\prime}(t), t \in[0, R]$ (and hence $u(t) \geq u_{0}(t), t \in[0, R]$ ).

Corollary 4.6. Assume that $f$ is continuous on $[0, R] \times \mathbb{R}^{r+1}$, that the hypotheses $\left(H_{2}\right)$, $\left(H_{4}\right)$ are satisfied, that the Lipschitz condition (4.2) is satisfied for all $\left(t, x_{1}, \ldots, x_{r+1}\right)$, $\left(t, y_{1}, \ldots, y_{r+1}\right) \in[0, R] \times \mathbb{R}_{+}^{r+1}$ with $x_{1} \geq y_{1}, x_{2} \geq y_{2}, \ldots, x_{r+1} \geq y_{r+1}$, and that the function $f(., 0, \ldots, 0)$ belongs to $(C[0, R])^{+}$. Then, Problem (1.8), $u \in C_{1}[0, R]$ has a unique solution with a positive derivative (and hence the solution $u$ is itself positive).

\section{Concluding remarks}

(1) The case $\alpha=1$ and $c \leq 1$ in Theorem 4.5 is the order counterpart of [15, Theorem 3]. Moreover, since there are many functions $f$ which satisfy the Lipschitz condition (4.2) without the standard one (4.1), we see the need of Corollary 3.4 instead of Theorem 1.2 to get a fixed point of the operator defined by (4.4). Indeed, as a simple example, consider the discontinuous function $f:[0, R] \times \mathbb{R}^{2} \rightarrow \mathbb{R}$ defined by

$$
f(t, x, y)= \begin{cases}\frac{1}{2} x+1 & \text { if } x>-1 \\ 1-x^{2} & \text { if } x \leq-1\end{cases}
$$

and let $h_{1}(t)=t$ for every $t \in[0, R]$. In this case, the null function on $[0, R]$ is a lower solution of Problem (1.8), $u \in A C[0, R]$, all the hypotheses $\left(H_{1}\right)-\left(H_{4}\right)$ are fulfilled, and Problem (1.8), $u \in A C[0, R]$ and $u^{\prime}(t) \geq 0$ for a.e $t \in[0, R]$ reduces to the simple initial value problem

$$
u^{\prime}(t)=\frac{1}{2} u(t)+1 \text { for a.e. } t \in[0, R], u(0)=0,
$$

which has a unique solution $u \in A C[0, R]$ with a positive derivative.

(2) On the other hand, the following easy situation illustrates the need of Corollary 3.4 instead of Theorem 1.1 or Theorem 1.3. Let $\mathbb{R}^{2}$ be endowed with their Euclidean norm and coordinatewise ordering. Let $T: \mathbb{R}^{2} \rightarrow \mathbb{R}^{2}$ be equal to $A=\left(\begin{array}{ll}0 & 1 \\ 0 & 0\end{array}\right)$. Clearly, all conditions of Corollary 3.4 are fulfilled and $(0,0)$ is the unique fixed point of $T$. In particular, the pair $T, A$ satisfies the contraction condition (1.7). However, it is easy to see that the contraction condition of Theorem 1.1 fails and that for any operator $B \in B^{+}\left(\mathbb{R}^{2}\right)$ with $\|B\|<1$, the pair $T, B$ does not satisfy the contraction condition of Theorem 1.3.

(3) Theorems 4.1 and 4.5 can be stated under slight suitable modifications if we assume the existence of an upper solution instead of a lower solution of Problem (1.8). 
(4) The monotone iterative sequences of approximate solutions for Problem (1.8).

This is for the case when this problem admits simultaneously a lower and an upper solutions $u_{0}$ and $v_{0}$ with $a \leq u_{0}^{\prime}(t) \leq v_{0}^{\prime}(t) \leq b$ for almost all (resp. for all) $t \in[0, R]$ and for some $a, b \in \mathbb{R}^{+}$. If the other hypotheses of Theorem 4.1 (resp. 4.5) hold true for both the lower and the upper solutions $u_{0}$ and $v_{0}$ (with the suitable modifications for the upper solution $v_{0}$ ) and if we keep the notations of the proof of Theorem 4.1, then the operator $T$ is now defined on the order interval $\left[z_{0}, w_{0}\right]$ of $L_{1}[0, R]$ (resp. $C_{1}[0, R]$ ), where $w_{0}$ is generated similarly from the upper solution $v_{0}$, with $z_{0}$ and $w_{0}$ as a lower and an upper fixed points, respectively. In this case, the latter two theorems provide a unique solution $u$ of Problem (1.8) with $u_{0}^{\prime}(t) \leq u^{\prime}(t) \leq v_{0}^{\prime}(t)$ for almost all (resp. for all) $t \in[0, R]$ (and hence $\left.u_{0}(t) \leq u(t) \leq v_{0}(t), t \in[0, R]\right)$. Define the sequences of functions on $[0, R]\left(f_{(n)}\right)$ and $\left(f^{(n)}\right)$ by $f_{(0)}(t)=z_{0}(t), f^{(0)}(t)=w_{0}(t)$, and inductively by

$$
\begin{aligned}
f_{(n)}(t) & =f\left(t, \int_{0}^{h_{1}(t)} f_{(n-1)}(s) d s, \ldots, \int_{0}^{h_{r}(t)} f_{(n-1)}(s) d s, f_{(n-1)}(t)\right), \\
f^{(n)}(t) & =f\left(t, \int_{0}^{h_{1}(t)} f^{(n-1)}(s) d s, \ldots, \int_{0}^{h_{r}(t)} f^{(n-1)}(s) d s, f^{(n-1)}(t)\right) .
\end{aligned}
$$

It follows easily from $f_{(n)}=T^{n} z_{0}, f^{(n)}=T^{n} w_{0}$, and Corollary 3.4 that the monotone sequences of functions $\left(\int_{0} f_{(n)}(s) d s\right)$ and $\left(\int_{0} f^{(n)}(s) d s\right)$ converge uniformly on $[0, R]$ to the solution of Problem (1.8) .

\section{References}

[1] C.D. Aliprantis and R. Tourky, Cones and Duality, Graduate Studies in Mathematics, Vol. 84, Amer. Math. Soc., Providence, Rhode Island, 2007.

[2] J.M. Borwein and D.T. Yost, Absolute norms on vector lattices, Proc. Edinb. Math. Soc. 27, 215-222, 1984.

[3] S. Carl and S. Heikkilä, Fixed Point Theory in Ordered Sets and Applications, Springer, New York, 2011.

[4] L.H. Erbe, W. Krawcewicz, and D. Guo, Positive solutions of two-point boudary value problems for nonlinear integro-differential equations in Banach spaces, Differ. Equ. Dyn. Syst. 2, 161-171, 1994.

[5] K.H. Förster and B. Nagy, On the local spectral radius of a nonnegative element with respect to an irreducible operator, Acta Sci. Math. (Szeged), 55, 155-166, 1991.

[6] D. Guo and V. Lakshmikantham, Nonlinear Problems in Abstract Cones, Academic Press, Inc., Boston, 1988.

[7] D. Guo, Multiple positive solutions of impulsive Fredholm integral equations and applications, J. Math. Anal. Appl. 173, 318-324, 1993.

[8] D. Guo, Y.J. Cho and J. Zhu, Partial Ordering Methods in Nonlinear Problems, Nova Science Publishers Inc., Hauppauge, 2004.

[9] R.D. Holmes and A.T. Lau, Nonexpansive actions of topological semigroups and fixed points, J. Lond. Math. Soc. (2), 5, 330-336, 1972.

[10] G.S. Ladde, V. Lakshmikantham and A.S. Vatsala, Monotone Iterative Techniques for Nonlinear Differential Equations, Pitman, Boston, 1985.

[11] Z. Liang, Some properties of nonlinear operators and positive solutions of a class of integral equations, Acta Math. Sinica (Chin. Ser.), 40, 345-350, 1997. 
[12] J.J. Nieto and R. Rodriguez-Lopez, Contractive mapping theorems in partially ordered sets and applications to ordinary differential equations, Order, 22 223-239, 2005.

[13] A.C.M. Ran and M.C.B. Reurings, A fixed point theorem in partially ordered sets and some applications to matrix equations, Proc. Amer. Math. Soc. 132, 1435-1443, 2004.

[14] C.A. Stuart, Positive solutions of a nonlinear integral equation, Math. Ann. 192, 119-124, 1971.

[15] M. Zima, A certain fixed point theorem and its applications to integral-functional equations, Bull. Aust. Math. Soc. 46, 179-186, 1992.

[16] M. Zima, Positive Operators in Banach Spaces and Their Applications, Wydawnictwo Uniwersytetu Rzeszowskiego, Rzeszow, 2005. 